\title{
Challenges and Applications of Wireless Body Area Networks
}

\author{
Rajeev Sharma, Sandeep singh kang
}

\begin{abstract}
Modified low-power, ultra-slim, light in weight, intelligent devices are the result of recent advances in technology. Wireless Body Area Network (WBAN) is a replacement technology that can be used to incorporate these devices \& thereby provide health monitoring applications in healthcare. Further development of wireless communications in recent years has led to the use of sensing element networks, which are low priced. These networks have a wide variety of applications. Various technical problems in these application areas are being resolved by researchers across the world. These sensing component networks play a significant role in healthcare. These networks have deep roots in various sectors viz; engineering, medicine\& science \& can show good performance even in harsh climatic conditions. Therefore, this paper provides an associated degree of exposure for the analysis and applications of wireless body area networks (WBAN's), and body sensor networks (BSN's). Apart from it, it addresses a wide variety of challenges in these technologies.
\end{abstract}

KEYWORDS: Wireless body area networks (WBANs), body sensor networks (BSNs), ultra-wideband (IEEE 802.15.3) \& ZigBee (IEEE 802.15.3), Sensor, personal digital assistant (PDA)

\section{INTRODUCTION}

Realization of Wireless Body Area Networks (BANs) is now possible due to the advent of radio frequency technology \& sensors. A typical WBAN comprises three types of devices: sensors, actuators and personal digital assistant (PDA) [13]. These networks can serve as thebuilding blocks to build networks in the future \& can further help in providing better approach toward health monitoring.

Revised Manuscript Received on June 15, 2019.

Rajeev Sharma, Sandeep singh kang :

Chandigarh University, Gharaun ,Mohali ,Punjab ,India
These networks have the ability to pass on information of respiration, blood heat, sound, vibration and other important signs. In a WBAN, biomedical sensors, deployed on a patient's body, report their measurements to a data sink, which often links to a healthcare institution through wide area networks, by wireless transmission in a single-hop or multi-hop fashion [14]. Non-invasive sensors fail to detect $\&$ analyze functional reading, which can be intercepted by the nearest devices, with a mobile phone as an entrance. ZigBee is meant to produce a protocol, which is durable \& cost-efficient, thereby providing high stability\& performance. This paper provides a summary of wireless body area networks (WBANs), their practical applications, challenges and issues related to their security. This paper provides a comparison with OSI Model and with TG6 (ask Cluster Half Dozen) security issues. A large number of open-problems \& challenges that are needed to be selfaddressed to create wireless BAN's \& ZigBee have been addressed in the paper.

An organizer or doctor starts traffic on-demand in order to get exact data, largely for the aim of diagnosis of the patient's health. After nodes go higher than a predefined threshold, they start sending emergency traffic which may be contained within one second. Moreover, this kind of traffic is amenable to change\& non-periodic. Usually thetraffic is the normal onewith no time criticality. Normal traffic includes treatment of the patient for diseases like cancer, disorders related to heart, neural systems etc. The conventional knowledge is processed by the arranger. The organizer holds a wakeup route, a main radio, and a bridge perform, all of them connected to a knowledge interface. The wakeup circuit is employed to produce on- demand and imperative scenario passage. The organizer is connected to servers for communication.

Published By:

Blue Eyes Intelligence Engineering \& Sciences Publication 


\section{Challenges and Applications of Wireless Body Area Networks}

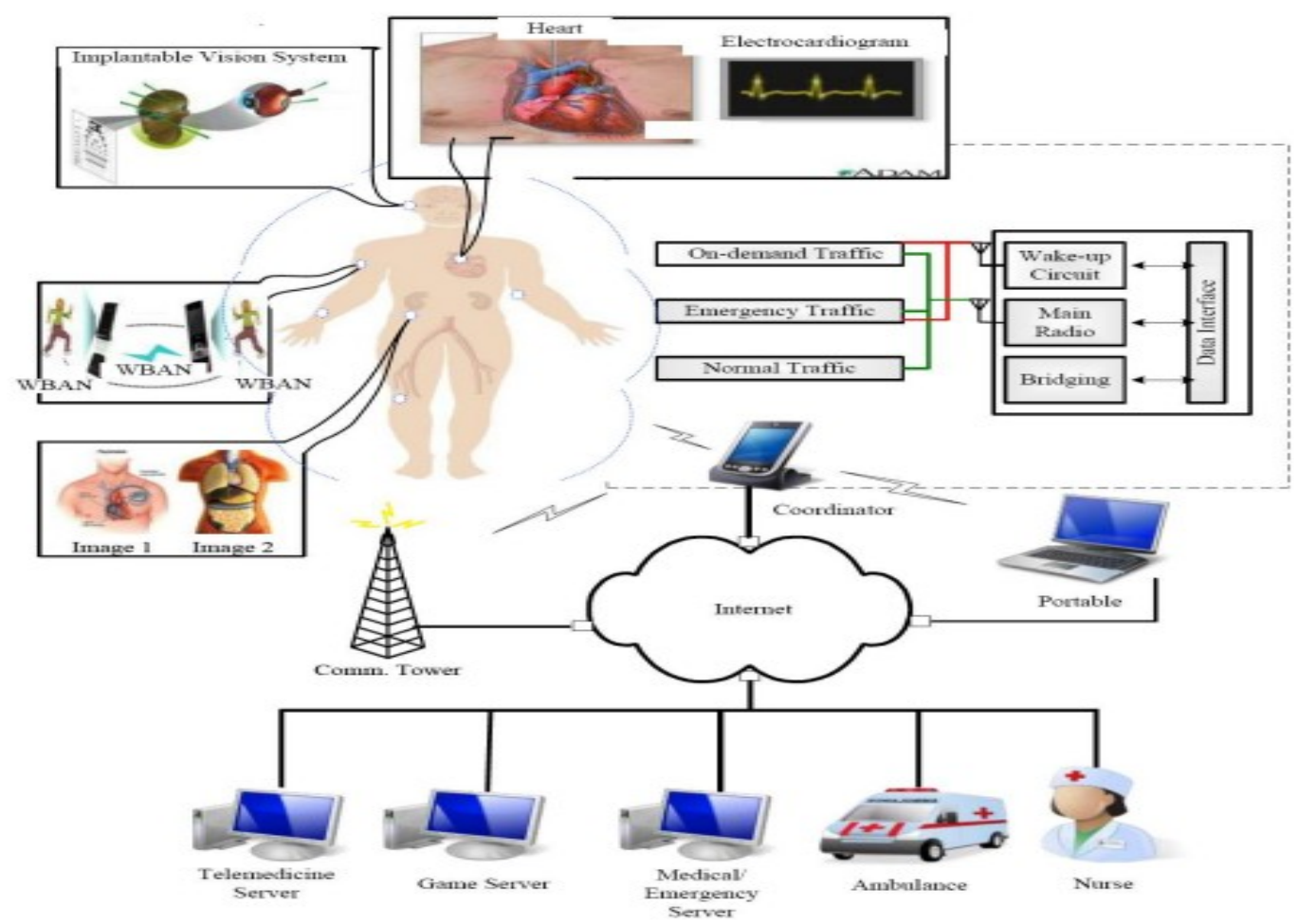

Figure 1 A WBAN design for application in the various areas of medical \& non-medical science [6]

Figure 1shows the WBAN traffic for medical \& other applications. A number of challenges have been introduced in the fields of administration, healthcare monitoring due to the rise of population inthe developed countries \& the rise of diseases in old aged people. Toprovide customized services to patients, there is an immense need for the use of wireless instruments to enhance performance.WBANsprovide an extremely reliable infrastructure for medical devices, particularly those ingrained inside the body. They consist of a variety of small sensors that may be fixed on the body as microchip, either beneath the skin or deeply

\section{A. E-Health Care}

WBANs provide interface for medical specialty, remote monitoring of human physiological knowledge. Patients will be treated remotely in near future. WBAN Provides healthcare application that offers immense contribution in improving patients healthcare and diagnosing and embeddedwithin the body tissues. This increases the capability of doctors to monitor the health of patients in a secure manner, thereby increasing the quality of life [1].Three communicationprotocols are applied by wireless BAN's [2], Bluetooth [3], ultra-wideband [4] \& ZigBee [5]. A wireless BAN is used to connect nodes that are implanted on the surface of the body for applications in healthcare, recreation, defense, or other areas. Following are the application of body area networks.

monitoring. Moreover, it provides medical rehabilitation and supervising of patients. Patients can now be examined at their homes while performing daily activities. 


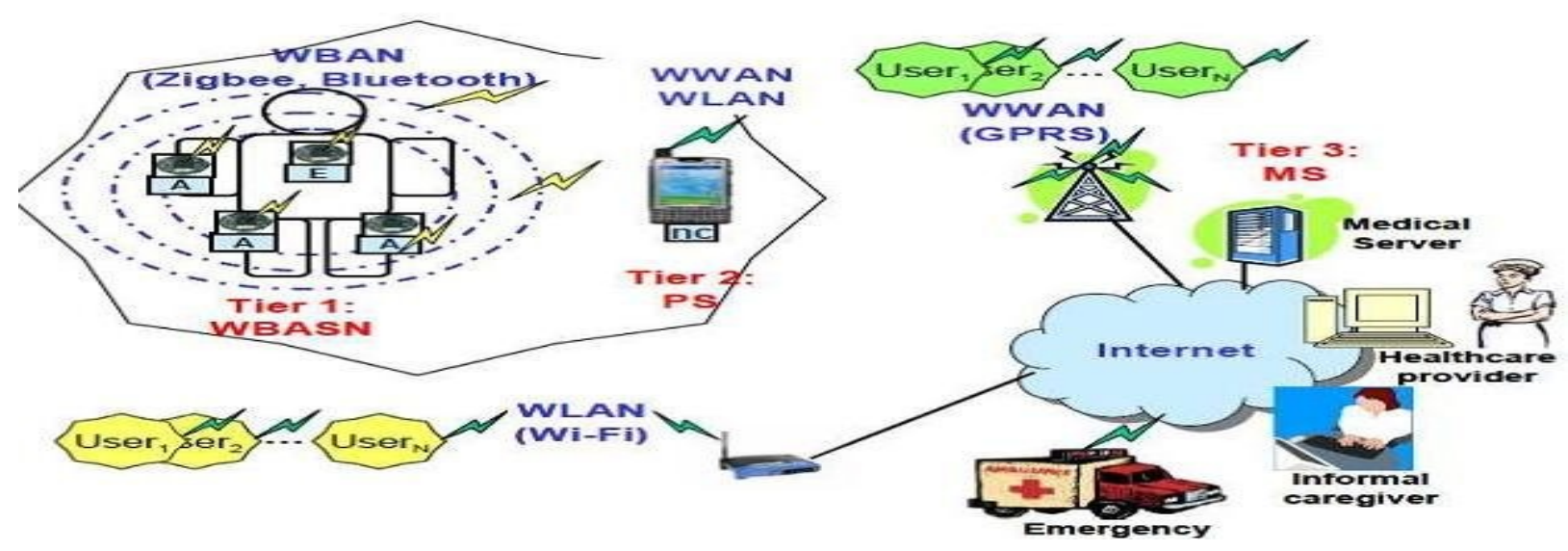

Figure 2 E-health care system designs [7]

\section{B. Lifestyle and Sports}

In [8], Wireless BAN has its utility in golf as well, wherein it resolves the problem of proper direction of the golf club. In addition to this supplying period response as per the limb of participant in terms of hip movement is also resolved. Conserving energy \&ability to perform at maximum altitude by the golf player corresponds to body sensory knowledge of player's movement. Not only does BAN provide better performance observation \& nipper observation in sports but it also extends its area to new functions for wireless body area networks.

\section{Military Applications}

Accomplished experiments are using auditory sensing elements to detect and limit short signals from mortars, weapon systems has been successfully carried out by ARL, the Army Research laboratory which will have a powerful consequence on deadliness \&survivability in battlefields [9].

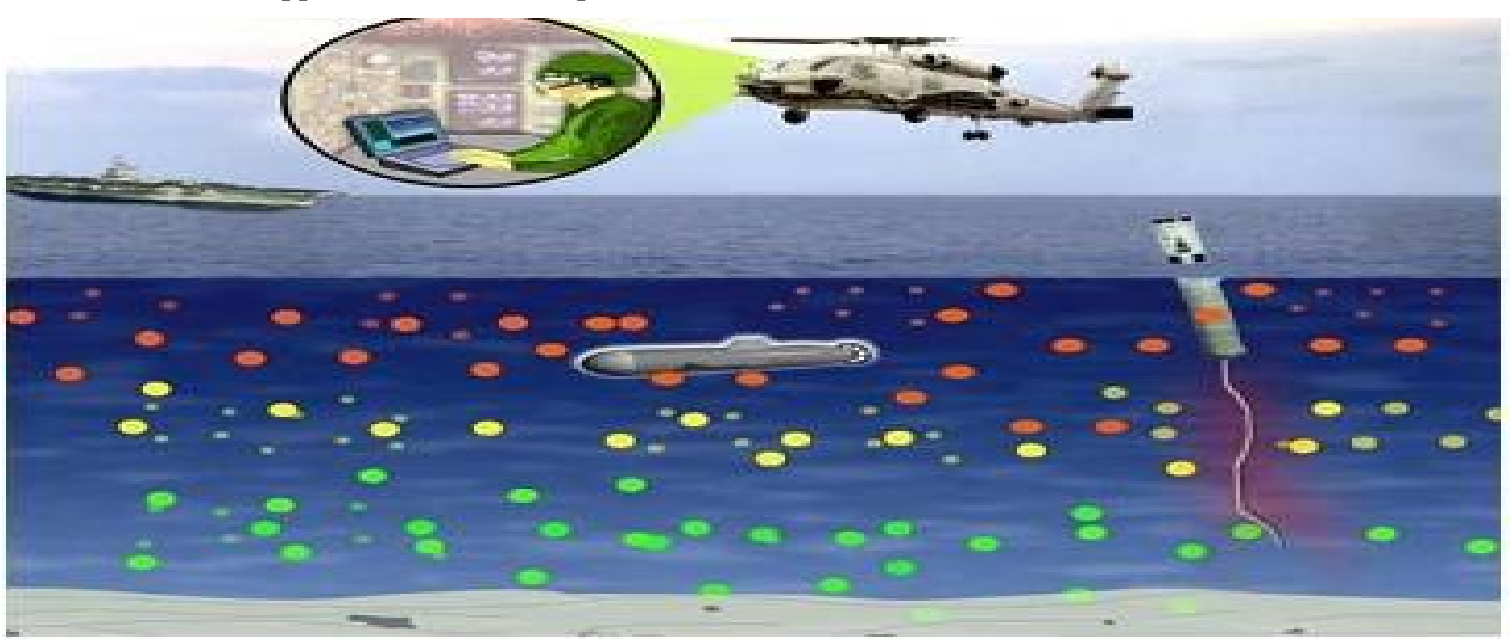

Figure 3WBAN actions in Military Operations [9]

Among the battlefield BAN's applicability is immense, which includes health monitoring, location based services\& detection of hot temperature. BAN combined with military uniform forms a wearable network connecting devices like PDA's,cameras,life support sensors, health monitoring which then proceeds to transfer knowledge to \& from the soldier's wearable laptop.Chemical recognition, prevention of victims from fire \& observation of a soldier's state are some other functions provided by the networks.

\section{WBAN for Animals}

Wireless BAN serves as an important tool for diagnosis of vapioushinfactious diseases in Blue Eyes Intelligence Engineering \& Sciences Publication 


\section{Challenges and Applications of Wireless Body Area Networks}

individuals \& animals. This is quite important because both animals \& humans share a symbiotic interdependent relationship.

\section{E. Preventing Cable and Conductor Theft}

Reports of cable stealing including both power cables and telecommunication lines have been reported.Different types of wires are being stolen dueto increasing price of metal. Business against Crime (BAC) African country declares that the knock-on effects to the economy is, conventionally, 10 times superior to the expenses needed for substituting stolen wire.

\section{F. Networking and communications}

These technologies decrease the prices of different resources which accordingly facilitate integration with theinternet.

\section{TECHNIQUES USED}

Various latest radio communication technologies are discussed in this paper.

\section{A. ZigBee and IEEE 802.15.4}

In order to implement BAN in various applicationareas, two interrelated technologies named ZigBee [10] and IEEE 802.15.4 provide a strong foundation. The previous standard enhances the IEEE 802.15.4 standard by adding networking related security layers and an application framework to provide complete wireless sensing element network (WSN) systems whereas the latter standard covers the physical (PHY) and medium access control (MAC) layers targeting low-rate short-range radio-communications, most suitable for BAN nodes. The ZigBeesupports technologies that enable systems with practical multi-vendor devices \& lowpower consumption, for instance, the ZigBee good Energy and Building Automation Profiles target applications within the realm of good energy use involving numerous forms of appliances within the home atmosphere, and building and industrial automation within the industrial atmosphere. In order to fulfill Continua Health Alliance necessities within the realm of health and fitness observation,ZigBee has proposed a ZigBee Healthcare Profile Technology. Whenever radio transmissions are done over ZigBeeband, transmissions suffer considerably from extremely variable path loss around the body [11]. This hinders data-rate, thereby hindering the support ZigBeeoffers forBAN applications.
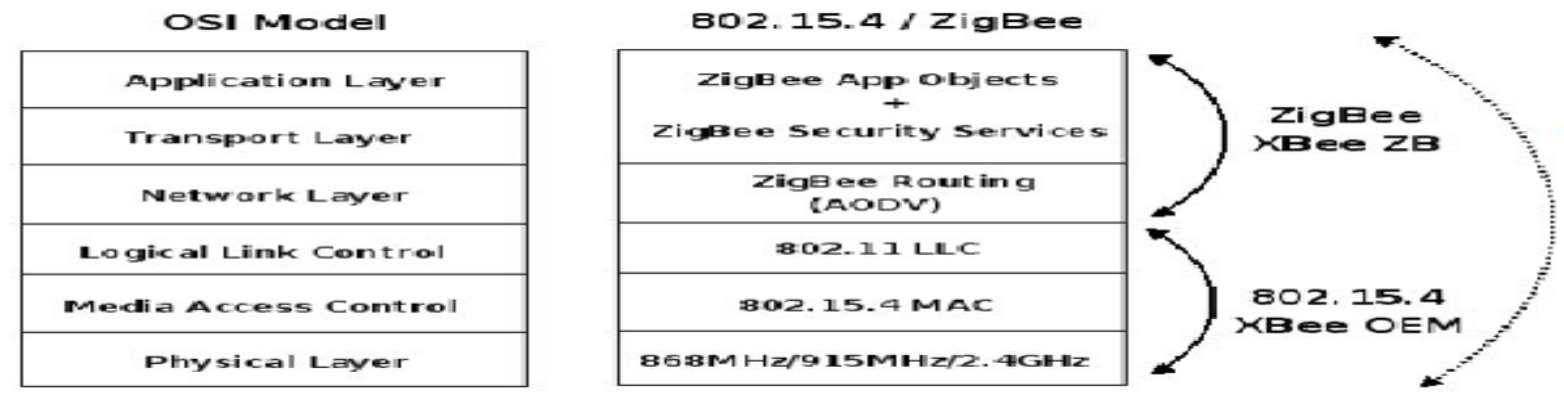

Figure 4 802.15.4/ZigBee comparisons with OSI Model [10]

\section{B. Bluetooth Low Energy}

Bluetooth is widely used for connecting a large number of private devices. This technology helped modify knowledge and voice communications. By forming a star like topology referred to as a piconet, these devices operate within the pair of.4 gigacycle functional system band and have the capability to access seventy nine channels [12]. This technology has a data rate of up to one Mbps\&utilizes fewer channels for pairing therebyincreasing the performance.

\section{Comparison of Techniques}

ZigBee and Bluetoothtechnologies have a lot in common, like each of them operates within the same waveband of 2.4 gigacycle and belong to a wireless personal area network (IEEE 802.15).There are a large number of variations between these two technologies.

Whereas Bluetooth eliminates cabling between shortdistanced devices,ZigBee is

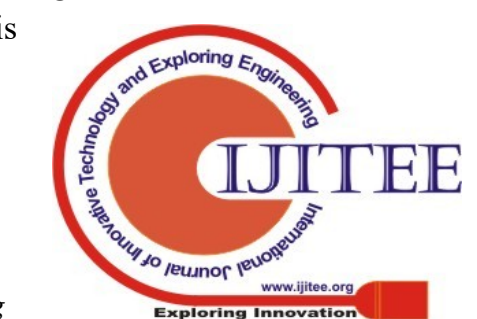


additionally home-bound towards high technology. Bluetooth eliminates the need of cables between nodes that arein the close vicinity. The users of this technology are always ready to exchange documents,files, images etc.

ZigBee is anticipated to be ready to eradicate the need of electrical cables in homes thereby permitting the liberty of wireless lightweight switches.

\section{Technical Parameters}

Bluetooth features a network range of one to one hundred meter, whereas for ZigBeeit isupto seventy meters.

Bluetooth batteries are rechargeable; however this is not the case for ZigBee, althoughZigBee batteries are long lasting.

1) ZigBee focuses on automation whereas Bluetooth focuses on connectingcell phones in close vicinity.

2) ZigBee networks support longer range devices whereas Bluetooth networks support short range devices.

3) ZigBee is suitable for critical applications (join time $=30$ milliseconds) whereas Bluetooth's longer join time is harmful (3 seconds).

\section{ROUTING PROTOCOLS IN WBAN}

Routing protocols in WBANs are classified into following five categories discussed as under:

\section{A. Cluster-based Algorithms}

The first category of routing protocols in WBANs is Cluster-based routing algorithms that divide nodes in WBANs into completely different clusters and assign a cluster-head for every cluster. Data is routed through the cluster-heads to the sink. These routing protocols decrease the amount of direct transmissions from the sensors to the bottom station but large delay\& overhead related to cluster choice are the disadvantages of these protocols.

\section{B. Probabilistic Algorithms}

Probabilistic routing protocols sporadically update their cost function based on link state data, and establish their path among routes with minimum cost.

\section{Cross-Layer Algorithms}

Cross layer routing protocols are low energy consuming protocols whichprovide high output, but in terms of performance they lag behind.

\section{Temperature-based Algorithms}

Radio signals generated via wireless communication generate electric and magnetic fields which leads to the rise in the temperature of the body. If the temperature inside the body continues to rise body tissues might be damaged \& this may further lead to blood flow reduction in some organs.

\section{E. QoS-based Routing Algorithms}

The last class is QoS routing protocols, which provide a standard approach by presenting separate modules for various QoS metrics that, operate in coordination with one another. The modules employed in this methodology are the reliability-sensitive module, the delay-sensitive module and also the neighbor manager.

\section{WBAN CHALLENGES}

Wireless BAN is an emerging technology withplenty of issues to be addressed. WBAN has to face anumber of challenges like confidentiality which is the most important issue. Some important technical challenges are discussed below:

1) Number of nodes: The number of nodes should neither be too many in number nor too less in number depending on the specific applicability.

2) Size of the node. This is an important challenge in WBANs.

3) Security of data: Various security provisions should be provided in WBANs to prevent the sensitive information.

4) Wirelesstools: There is an increasing demand for supply of low power wireless tools in WBANs.

5) Compatibility: The various types of equipment's like sensors etc. used in WBANs should be compatible with each other, otherwise sensors supplied externally might increase cost.

\section{SECURITY IN WBAN}

Before instigating encoding, the protection strategy should

Published By:

Blue Eyes Intelligence Engineering \& Sciences Publication 


\section{Challenges and Applications of Wireless Body Area Networks}

be considered. Wireless Body area Networks (WBAN) are used to diagnose various diseases in patients .WBAN provides three levels of security viz level 1 , level2 \& level 3.Level 1 has no inbuilt mechanism for security. Level2 provides a mechanism for authentication only whereas level 3 provides a mechanism for encryption as well as authentication of data.

\section{A. Data Confidentiality \& Integrity}

Data confidentiality in WBAN's is the most important issue that needs to be addressed. It avoids information leakage. Knowledge integrity is equally important. Importinformationof the patient could be lost \& this could be detrimental from a privacy perspective.

\section{B. Data Authentication}

Authentication helps establish proof of identities.MAC (Message Authentication Code) computed using secret key can ensure data authentication.

TABLE I

Security methods in communication protocols

\begin{tabular}{|l|l|l|l|}
\hline Protocols & Bluetooth & ZigBee & TG6 \\
\hline Confidentiality & Yes & Yes & Yes \\
\hline Integrity & No & No & Yes \\
\hline Non repudiation & No & No & No \\
\hline Authentication & Yes & Yes & Yes \\
\hline Authorization & No & No & No \\
\hline
\end{tabular}

\section{Data Freshness and Secure Localization}

Data needs to be refreshed time \&again. Old data is stale\&useless. Data freshness has two type'sviz strong freshness wherein data is sent quickly with less delay \& weak freshness wherein only partial data is sent with some delay.

\section{Availability andSecure Management}

In healthcare, it is very important to respond to a patient quickly, supply him the medicines etc. Thus there should be systems that are available $24 / 7$ for a fast response .If any node of the network is attacked, to ensure response to the patient; the compromised node should be changed to ensure secure management $\&$ high availability.

\section{CONCLUSION}

The aim of this paper is to study an emerging technology called Wireless Body Area Networks (WBANs).This paper also studies technologies likeultra-band,ZigBee,and Bluetooth. In addition to it, an insight into the challenges, applications \& security issues associated with WBANs \& different ways to tackle the various security issues are discussed.

\section{REFERENCES}

[1] Ragesh, G. K., \&Baskaran, K. "An Overview of Applications, Standards and Challenges in Futuristic Wireless Body Area Networks", Journal of Computer Science, (2012), 9(1), 180-186.

[2] E. M. Staderini, -UWB radars in medicine,IEEE Aerospace and Electronic Systems Magazine, vol. 17(1), pp. 13-18, 2002.

[3] IEEE standard for information technology-telecommunications and information exchange between systems - local and metropolitan area networks - specific requirements part 15.1: wireless medium access control (MAC) and physical layer (PHY) specifications for wireless personal area networks (WPANs),॥ IEEE Std 802.15.1TM, 2005.

[4] IEEE standard for information technology - telecommunications and information exchange between systems-local and metropolitan area networks - specific requirements part 15.3: wireless medium access control (MAC) and physical layer (PHY) specifications for high rate wireless personal area networks (WPANs) amendment 1: MAC sublayer,l IEEE Std 802.15.3b, 2006.

[5] IEEE Standard for Information technology - telecommunications and information exchange between systems - local and metropolitan area networks - specific requirements part15.4: wireless medium access control (MAC) and physical layer (PHY) specifications for low-rate wireless personal area networks (LR-WPANs),॥ IEEE STD 802.15.4TM, 2006.

[6] S. U., Khan, P., Ullah, N., Saleem, S., Higgins, H., \&Kwak, K. S. A Review of Wireless Body Area Networks for Medical Applications.Sciences-New York, 1-7(2009).

[7] C. Scott, G. Heath and J. Svoboda (2006, April) Preprint: -Vibration monitoring of power distributionpoles.|[[Online].http://www.inl.gov/technicalpublications/Docu ments/3493254.pdf.

[8] Kyung Sup Kwak1, M. A. Ameen1, Daehan Kwak1, Cheolhyo Lee2, Hyungsoo Lee2, A Study on Proposed IEEE 802.15 WBAN MAC

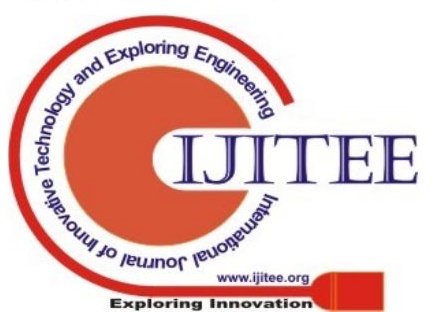


[9] Y. Xiao, X. Shen, B. Sun, L. Cai, Security and privacy in RFID and applications in telemedicine, IEEE Communications Magazine, 44(4) 6472(2006).

[10] ParneetDhillon, HarshSadawarti, "A Review Paper on Zigbee (IEEE 802.15.4) Standard". International Journal of Engineering Research \& Technology (IJERT) IJERT ISSN: 2278-0181, Vol. 3 Issue 4, April - 2014

[11] IEEE Standard 802, Part 15.4: Wireless Medium Access Control (MAC) and Physical Layer(PHY) specifications for low-rate wireless personal area networks (WPANs), IEEE Std 802.15.4d $\mathrm{d}^{\mathrm{TM}}$-2009, IEEE Computer Society, 17 April 2009.

[12] ArwaKurawar, AyushiKoul, Prof. Viki TukaramPatil, "Survey of Bluetooth and Applications", International Journal of Advanced Research in Computer Engineering \& Technology (IJARCET) Volume 3 Issue 8, August 2014

[13] Rongrong Zhang, Amiya Nayak, \&Jihong Yu. "SleepScheduling in Energy Harvesting Wireless Body Area Networks", IEEE Communications Magazine, February 2019, 95-101.

[14] Yuan-Yao Shih, Member, IEEE, Pi-Cheng Hsiu, Senior Member, IEEE, and Ai-Chun Pang, Senior Member, IEEE, "A Data Parasitizing Scheme for Effective Health Monitoring in Wireless Body Area Networks", IEEE, 1536-1233 (c) $2018,1-14$.

\section{AUTHORS PROFILE}

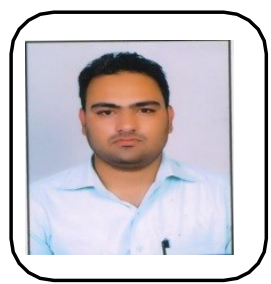

Rajeev Sharmareceived his B.Tech in Computer Science Engineering from Deshbhagat Engineering College Mandigobindgarh (PTU) in 2008 and M.Tech in Computer Science and Engineering from Gura Nanak Dev Engineering College (PTU) in 2015. He has teaching experience of 12 years. He has published various research papers and his area of interest is computer Networks and mobile communications. He is currently pursuing Ph.D. degree in Computer Science and Engineering from Chandigarh University, Gharuan, Mohali (Punjab).

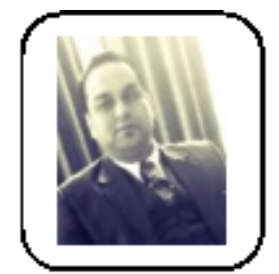

Dr. Sandeep Singh Kang is working as Professor in Department of Computer Science \& Engineering at Chandigarh University.Gharuan, Mohali (Punjab). He did his B.tech, M.Tech and $\mathrm{Ph} . \mathrm{D}$ in Computer Science \& Engineering. He has published more than 70 research papers and one book. He has 15 Years Teaching experience in various colleges and universities. He is the life member of ISTE
Published By:

Blue Eyes Intelligence Engineering \& Sciences Publication 